\title{
Diseño de un cultivador hidropónico vertical para uso doméstico
}

\section{Design of a vertical hydroponic cultivator for domestic use}

\author{
Daniel González, Yirene Caballero ${ }^{1}$, Orlando Aguilar ${ }^{2 *}$ \\ ${ }^{1}$ Licenciatura en Ingeniería Mecánica, Universidad Tecnológica de Panamá, ${ }^{2}$ Facultad de Ing. Mecánica, Universidad \\ Tecnológica de Panamá. Grupo de Investigación en Energía Renovable y Ambiente (GIERA)
}

\begin{abstract}
Resumen En los últimos años la hidroponía ha sido una técnica que ha demostrado ser eficiente para la producción de hortalizas y legumbres. El presente trabajo pretende detallar la construcción de un cultivador hidropónico vertical para espacios domésticos utilizando semillas de lechuga. Esto pretende beneficiar a personas que deseen realizar su propio huerto de lechuga en el hogar y que no cuenten con terreno o condiciones ambientales tradicionales para realizarlo. Se va a construir el equipo a base de tuberías de PVC, mangueras, aspersores de riego por goteo, bombas de agua y aire para peceras, que permita oxigenar el agua, temporizadores y luces LEDs, para compensar la función de la luz solar. Las semillas irán germinando y creciendo en el cultivador gracias a sustratos que proporcionarán los nutrientes, manteniendo una correcta circulación del agua para que se mantenga húmedo el sustrato. Se busca tener una producción de lechuga a bajo costo, que sea continua y abundante a lo largo del año en comparación al proceso tradicional de cultivo; rico en nutrientes, libre de pesticidas, de fácil y rápido acceso para todo público.
\end{abstract}

Palabras clave Cultivo, hidroponía, lechuga, nutrientes, raíces, sustratos.

\begin{abstract}
In recent years hydroponics has been a technique that has proven to be efficient for the production of vegetables and legumes. The present work intends to detail the construction of a vertical hydroponic cultivator for domestic spaces using lettuce seeds. This aims to benefit people who want to make their own lettuce garden at home and who do not have land to do so. The equipment will be built with PVC pipes, hoses, drip irrigation sprinklers, water and air pumps for fish tanks, which allow the water to oxygenate, timers and LED lights, to compensate for the function of sunlight. The seeds will germinate and grow in the cultivator thanks to substrates that will provide the nutrients, maintaining a correct circulation of the water so that the substrate remains moist. The aim is to have a low-cost production of lettuce, which is continuous and abundant throughout the year compared to the traditional process of cultivation, rich in nutrients, free of pesticides, easy and quick access for all public.
\end{abstract}

Keywords Cultivation, hydroponics, lettuce, nutrients, roots, substrates.

* Corresponding author: orlando.aguilar@utp.ac.pa

\section{Introducción}

La palabra "hidroponía" se compone por los vocablos "hidro", lo cual significa agua, y "ponía", que significa labor o trabajo. El cultivo hidropónico es aquel que prescinde totalmente de la tierra para cultivar los alimentos. Este tipo de cultivo evita algunos problemas como la necesidad del uso de plaguicida, por lo que se obtiene un control casi total sobre el entorno, lo cual se traduce en cultivos más saludables. Un huerto hidropónico funcionará con las raíces en suspensión y con soportes variados. Las raíces absorben una solución balanceada de nutrientes disuelta en agua que cumple con todos los requisitos necesarios para el desarrollo de la plantación. El cultivo hidropónico podría ser una oportunidad para personas que, por falta de espacio y condiciones ambientales adecuadas, no pueden tener su propio huerto. De igual forma, este método puede introducirse en la producción a nivel industrial, para poder producir en gran medida los cultivos de hortalizas y legumbres que se desea.
La hidroponía permite producir plantas principalmente legumbres y hortalizas, en estructuras simples o complejas, aprovechando sitios o áreas como azoteas, suelos infértiles, invernaderos climatizados o no, pero principalmente espacios interiores, considerando el no uso de suelo en el cultivo. A partir de este concepto se desarrollaron técnicas que se apoyan en sustratos (medios que sostienen a la planta), o en sistemas con aportes de soluciones de nutrientes estáticos o circulantes, sin perder de vista las necesidades de la planta como la temperatura, humedad, agua y nutrientes. El estudio de los macronutrientes (elementos químicos como nitrógeno, fósforo, potasio, azufre, calcio, etc.) ayudó en gran medida a perfeccionar la hidroponía hasta poder realizar cosechas comerciales a gran escala [1]. Con la técnica de cultivo sin suelo es posible obtener hortalizas de excelente calidad y sanidad, permitiendo usos más eficientes del agua, nutrientes y espacio físico.

No es una metodología moderna para el cultivo de plantas, sino una técnica ancestral. En la antigüedad hubo culturas y 
civilizaciones que utilizaron esta metodología como medio de subsistencia. El desarrollo actual de la técnica de los cultivos hidropónicos está basado en la utilización de mínimo espacio, mínimo consumo de agua y máxima producción y calidad [2].

En el mundo, las ciudades han crecido y siguen aumentado su densidad poblacional. Debido a este crecimiento y a la reducción de zonas verdes en las ciudades, se deben buscar alternativas de producción que permitan obtener alimentos, plantas medicinales y ornamentales, en espacios reducidos, con pocas posibilidades de siembra y con recursos limitados de riego. Adicionalmente, con el aumento del consumo de alimentos orgánicos, también se hace importante, el poder contar con métodos de producción que permitan disminuir al máximo el uso de fertilizantes e insecticidas de fuentes químicas, remplazándolas por agentes orgánicos.

Los cambios en los tipos de construcción, principalmente los de vivienda, los cuales han pasado de casas amplias, con patios y antejardines, por apartamentos estrechos, con pequeñas terrazas, pueden presentar un problema para realizar un cultivo en espacios cerrados. De igual modo, los parques, colegios, algunas universidades, así como edificios del gobierno, han venido disminuyendo sus zonas verdes, por zonas en concreto para el mejor aprovechamiento económico del espacio y, en algunos casos, por cuestiones de salubridad pública, que hacen necesario implementar dispositivos que permitan traer la naturaleza a las ciudades. Sin embargo, la velocidad con la que se mueve el mundo moderno, los compromisos, los desplazamientos, etc., hacen que se dificulte el mantenimiento y sostenimiento de estas zonas en las ciudades.

Por lo anterior, se vuelve una alternativa viable, en las ciudades, la incorporación de sistemas de cultivos hidropónicos, con sistemas autónomos de riego que permitan a los propietarios la sostenibilidad del sistema, con una atención básica y periódica [3].

Mediante este proyecto se espera diseñar y construir un sistema de cultivo hidropónico para uso doméstico, el cual se utilice para cultivar lechuga u otros vegetales para uso ornamental y/o consumo.

\section{Metodología}

Para la confección del cultivador hidropónico vertical doméstico, se utilizaron materiales de fácil y económica adquisición, tales como tuberías de PVC, bombas de agua y oxigenación de peceras, mangueras y aspersores de riego por goteo. Se procedió a cortar una tubería de PVC de $48^{\prime \prime}$ de longitud y 6" de diámetro. A partir de 8" de la base, se perforaron a lo largo de la tubería de PVC, tres líneas de cinco orificios de $1-7 / 8$ " de diámetro cada uno. Los mismos estan separados 8 " entre centros cada uno, y las tres líneas de orificios se encuentran separadas a $120^{\circ}$ entre sí. Bajo estas condiciones, se contó con un total de 15 orificios alrededor de toda la tubería, en los que se puede cultivar igual número de lechugas de manera simultánea. En cada uno de los orificios se

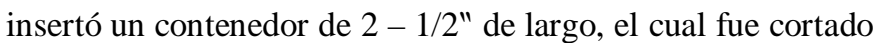
de manera transversal a $30^{\circ}$ con respecto a la horizontal superior hasta la primera $1-1 / 2^{\prime \prime}$ de largo, el corte se realizó de esta manera para garantizar la acumulación del agua en la contraparte del contenedor con respecto a la parte donde se hizo el corte.

Se procedió a implementar el uso del sistema NFT (Nutrient Film Technique) para el sistema de irrigación. El sistema NFT representa una técnica que impulsa por medio de una bomba, a través de una serie de tuberías, una lámina fina de solución nutritiva que se pone en contacto con las raíces de la planta colocada en un recipiente con una ligera pendiente, que facilita la recirculación del líquido [4]. El sistema de irrigación está compuesto de una bomba de agua de pecera, un temporizador, marca Aqualín, para que se realice un riego cada 30 minutos. Se incluye también, una bomba aire, marca Power Air Pump modelo AP-308, con el objeto de mantener el agua de circulación oxigenada, fundamental para el correcto desarrollo de la planta. Las propiedades minerales y fertilizantes del agua serán medidas haciendo uso de un medidor de $\mathrm{pH}$, un medidor de sólidos suspendidos totales y un medidor de conductividad eléctrica. De esta manera, se ajustan y mantienen los niveles de nutrientes según demandas de las plantas cultivadas.

Una vez construido el cultivador hidropónico, se procedió con su prueba, para lo cual, se seleccionaron y adquirieron las semillas de las variedades de lechuga para cultivo hidropónico.

En una bandeja de alveolos se colocó el sustrato y en él las semillas, tres por cada semillero, con el objetivo de que al menos, una de ellas germine. Se debe mantener toda la bandeja constantemente con agua, cerciorándose diariamente que el sustrato se mantenga húmedo de manera uniforme. Al cabo de una semana, las plántulas deben tener un tamaño de unos seis centímetros de altura y unas tres hojas por tallo [5]. Posteriormente, las plántulas son trasplantadas en los contenedores colocados para tal fin en el cultivador hidropónico y se continúa con el proceso de cultivo.

Se procede a verificar el $\mathrm{pH}$ que se suministra en la solución de nutrientes. Se ha observado que un $\mathrm{pH}$ óptimo para el crecimiento de la lechuga se encuentra entre los 6.2 y 6.5 [6]. Este rango es verificado por medio de un medidor $\mathrm{pH}-02$ Pentype. De igual forma, se mide la conductividad eléctrica por medio del equipo TDS\&EC meter(hold), donde los mejores resultados oscilan entre 2 y $3 \mathrm{ds} / \mathrm{m}$ para mantenerse en un valor recomendado entre los 1.5 y $2 \mathrm{dS} / \mathrm{m}$ [7].

\section{Resultados esperados}

En un cultivo hidropónico las semillas germinan, crecen y se desarrollan hasta su producción en un sustrato en contacto con una solución que contiene los nutrientes requeridos. El sustrato es el encargado de proveer el crecimiento de las plantas, en este caso lechuga, lo cual va a garantizar una elevada productividad y una reducción en los costos de producción [6]. El agua es recirculada, por lo tanto, no se va a 
presentar desperdicio, con un óptimo uso del agua acompañado de sus nutrientes. Muchos de los sustratos pueden ser de origen natural (turbas, virutas o aserrín), de origen mineral (arena o perlita) y de origen industrial (fibracel, lana de roca). Para poder definir uno que pueda ser elegido como el ideal a ser utilizado va a depender del uso específico al que vaya a ser destinado, esto quiere decir para la germinación de la planta, o crecimiento y desarrollo.

Al implementar la hidroponía vertical se puede tener su propio cultivo casero en cualquier espacio que se requiera en el hogar, tomando en cuenta que puede ser además de un sistema que produzca alimento. De igual forma este tipo de sistema de cultivo puede ser utilizado para la decoración de interiores que puede presentar una vivienda.

De obtener resultados favorables en el cultivo de lechuga en ambiente controlado en la ciudad de Panamá, se continuará con mayor investigación con el objeto de evaluar las mejores prácticas de cultivo y optimizar los rendimientos, considerando las condiciones ambientales de la ciudad de Panamá, en cuanto a temperatura y humedad relativa del aire, así como los requerimientos de iluminación artificial y temperatura controlada en ambientes interiores.

Gran parte de la luz solar que reciben las plantas se transforman en calor y parte de esta luz es aprovechada por ella para lograr un correcto crecimiento. Como parte de este proceso se propone utilizar luz artificial por medio de LEDs, ya que en muchas de las plantaciones hechas por cultivo hidropónico se utiliza este sistema para reemplazar la luz solar, además de ayudar a la plantación a la asimilación del carbono, la temperatura de las hojas, el balance hídrico, expansión de las hojas, el crecimiento de los órganos y tejidos, principalmente los tallos y su curvatura [8].

Con este proyecto se espera que los futuros usuarios puedan cultivar lechuga a lo largo de todo el año, haciéndolo en etapas, cosechando y plantando nuevas semillas, de forma tal que siempre estén presentes en uno o más cultivadores, lechugas en diferentes etapas de crecimiento. Esto representa una gran ventaja en comparación con los métodos tradicionales de cultivo, en los cuales, se requiere crear una planificación de los cultivos en campo abierto, para que el crecimiento de la lechuga coincida con el tiempo climatológico favorable para su correcto desarrollo [9].

\section{Conclusiones}

- La hidroponía es un sistema de cultivo fácil de mantener en lugares con poco espacio, ya que el mismo no presenta complicaciones en ninguna de las etapas de desarrollo de la planta, desde la siembra de las semillas hasta el cultivo de la legumbre.

- Con la continua implementación de estos equipos de cultivo se obtienen plantas de lechuga, con múltiples beneficios, como mayores nutrientes, mejor sabor y sin ningún tipo de pesticidas agregados en el proceso.
- El sistema hidropónico es un proyecto ideado para una producción continua a lo largo de todo el año.

- El rendimiento esperado de la producción por $\mathrm{m}^{2}$ de suelo utilizado es con creces, superior al rendimiento por $\mathrm{m}^{2} \mathrm{del}$ cultivo tradicional en suelo.

- Los costos de producción y transporte se espera que sean menores que en el método de cultivo tradicional, dado que puede cultivarse en o cerca de los centros de consumo.

\section{REFERENCIAS}

[1] María del Pilar Longar Blanco, María del Pilar M. Pérez Hernández, Edson Ríos Martínez, "El estado de Técnica de la Hidroponía", Revista Mexicana de Ciencias Agrícolas, Vol. 4, No. 5, agosto 2013.

[2] José Beltrano, Daniel O. Giménez, "Cultivo en Hidroponía", La Plata: Universidad de la Plata, 2015.

[3] Ricardo A. Forero R., Hollman G. Parra, Ricardo A. Luna C., Edwin R. Rivera C., "Agricultura Urbana: Sistema de Implementación de Cultivos Hidropónicos", Vol. 4, No. 1, noviembre 2011.

[4] Rogelio Armando Buendía Muciño, Ariel Cedillo Mendieta, Gabriel Vega Gutiérrez, "Diseño de un Invernadero Automatizado con Cultivo Hidropónico Vertical de Lechuga en El Rosario, Cuautitlán, Estado de México”, Instituto Politécnico Nacional, México D.F., septiembre 2012.

[5] Edson Ríos Martínez, "Hidroponia y Eco-innovación. Alternativas Tecnológicas para el Fomento de Huertos urbanos en la Ciudad de México”, México D.F., junio 2013.

[6] Gustavo Adolfo Ramírez Guzmán, "Sistema de Producción Hidropónica de Lechuga", Universidad Nacional Agraria La Molina, Perú, 2016.

[7] Penélope de La Rosa Topete, Irving Alberto Herrera Velásquez, "La Producción Hidropónica ¿Una alternativa Alimentaria en Espacios Urbanos?", Universidad Autónoma del Estado de México, México, mayo 2015.

[8] María Gabriela Bustamante Martínez, "Estudio de los Efectos de la Luz LEDs Sobre la Producción en Dos Variedades de Lechuga (Lactuca sativa L.) en un Sistema Hidropónico NFT Vertical”, Universidad de Guayaquil, 2016.

[9] Rogelio Armando Buendía Muciño, Ariel Cedillo Mendieta, Gabriel Vega Gutiérrez, "Diseño de un Invernadero Automatizado con Cultivo Hidropónico Vertical de Lechugas", Instituto Politécnico Nacional, México, septiembre 2012. 